\title{
Two methods for simulating mud discharge after emergency disconnection of a drilling riser
}

\author{
Chaowei Li", Honghai Fan, Zhiming Wang, Rongyi Ji, Weiyan Ren, Xu Feng \\ College of Petroleum Engineering, China University of Petroleum, No. 18 Fuxue Road, Changping, Beijing 102249, China
}

\begin{abstract}
In marine floating drilling, emergency disconnection of a drilling riser is required in harsh environments or loss of dynamic positioning control. After disconnection, drilling mud in the riser discharges directly from the riser bottom, and seawater refills the vacancy emptied out after mud falling through refill valves. This paper presents two new simulation procedures for this unsteady flow. The first one is a whole fluid column(WFC) model and it is solved by a cubic equation. The second one is a computational fluid dynamics(CFD) procedure, in which two high-resolution CFD schemes are applied for the first time to discrete a special governing equation, and Level Set method is adopted to track the interface between mud column and refilled seawater at each time step. Two methods can respectively provide variations of flow velocity, fluid pressure, whole column weight and flow friction force in a whole mud release duration. In particular, WFC method can easily predict overall trends of several parameters during a whole discharge period, which are prerequisite parameters for dynamic analysis of a riser in hanging state; CFD method is very sensitive to every detailed fluctuation of velocity and pressure in the initial moment of mud discharge, and can be integrated into a structural model for riser recoil response analysis. For a drilling riser with $2150 \mathrm{~m}$, it takes $195.83 \mathrm{~s}$ to replace all mud column by seawater, and the maximal discharge velocity is $14.61 \mathrm{~m} / \mathrm{s}$. During mud falling, the top of mud column keeps static for $2.29 \mathrm{~s}$ before the first pressure wave reaches, and fluid pressure of part column section drops to zero and lasts 1-3 s. In addition, the maximal values of fluid weight-loss and friction force are both close to half of the whole column weight. These results are beneficial for riser system design and risk control of riser recoil.
\end{abstract}

Keywords: drilling riser; emergency disconnection; mud discharge; riser recoil; Level Set method.

\section{Introduction}

In offshore drilling, emergency disconnection of a drilling riser from lower marine riser package(LMRP) is occasionally required in harsh environments or loss of dynamic positioning control. During an emergency disconnection sequence(EDS), drill pipe is sheared off instantly by a subsea blowout preventer(BOP) while drilling riser disconnected at LMRP(Paula and Fonseca, 2013). Because retaining drilling mud in a riser would magnify the riser dynamic response and time is limited to replace all mud with seawater before a sudden disconnection, drilling mud mostly releases freely from the riser bottom whilst the riser recoiling upwards(Miller et al., 1998). Mud discharge during riser recoiling is neither feasible to measure exactly in any real situation, nor easy to do experimental simulation. Hence, numerical simulation is an indispensable choice to obtain required parameters for riser recoil response analysis and structural dynamic analysis of a riser in hanging state.

Actually, efforts have been continuously made to achieve a more precise prediction of this unsteady flow. Yong et al.(1992) calculated falling velocity and column length for the first time during mud column falling from an open ended riser, and they done this via an initial "slug column model" without any water refilling. Miller et al.(1998) simulated the flow process by a time-domain program named "STARR" and "method of characteristics(MOC)". Lang et al.(2009) simulated mud release through one-dimensional Euler equations and a finite volume(FV) model, but did not provide enough description of this FV program for reference. Grytoyr et al.(2011) emphasized the influence of mud column on total weight and friction loads during riser recoil, and introduced a modified "slug column model" with considering a refill valve at riser top. Li et al.(2012) discussed the effect of mud shedding on riser recoil response and utilized Herschel-Bulkley rheology model for flow friction computation.
Although the "slug column model" can be easily used to calculate a rough trend of mud release in a whole discharge period, it only adopts constant velocity and acceleration of a single mud column in each time step, and thus can't provide fluctuation details in the first few seconds after disconnection. MOC can simulate some detailed variation of flow velocity and fluid pressure, but its fixed marginal grids are difficult to fit the boundary change and hard to capture the moving of upper mud level during mud release. Moreover, these previous papers did not provide either a governing equation or a specific solve method of these models/methods, and the published papers often considered a simplified pipe flow model without a drilling pipe in riser rather than a more practical annulus flow model.

This paper develops two new procedures. The first one is a "whole fluid column(WFC)" model, in which both drilling mud and refilled seawater are calculated; and we present a specific governing equation as well as a fresh algorithm by a cubic equation. The second one is a CFD procedure, in which for the first time, two high-resolution CFD schemes are applied to solve a governing equation and Level Set method is utilized to track the top mud level during fluid falling.

Especially, this paper considers an annulus flow model in the two methods and treats friction coefficients in a serious way. A case exhibits the fluctuations of flow velocity, fluid pressure and whole fluid weight, reveals the risk of pressure attenuation, and demonstrates the influence of friction force and column weightlessness induced by falling acceleration. The methods and case results may be beneficial for riser recoil analysis and riser system design.

\section{Assumptions and parameters statement}

When a riser is suddenly disconnected from LMRP in an emergency situation, a drill pipe string in riser is cut off by BOP at the same time. Once the riser bottom is opened, drilling mud releases 
instantly and seawater from refill valves replaces the vacancy emptied out by mud falling(Fig. 1).

To begin with, some basic hypotheses are given as follows:

(1) The flow channel of riser annulus is regular and its roughness is neglected.

(2) Refill valves locate at the riser top near sea level and are fully opened to jet enough seawater into the riser; drilling mud and seawater fills whole cross-section of riser annulus at every depth.

(3) Velocities, accelerations and pressures in this paper are average values at each cross-section of flow channel.

(4) Because the part of riser above sea level is very short when comparing with the whole riser length in seawater, the length in the air is neglected.

(5) Although riser would recoil upwards during mud falling, vertical shift of riser is ignored, and the flow channel length is fixed in current discussion.

(6) After disconnection, the bottom end of flow channel is completely opened without any throttle.

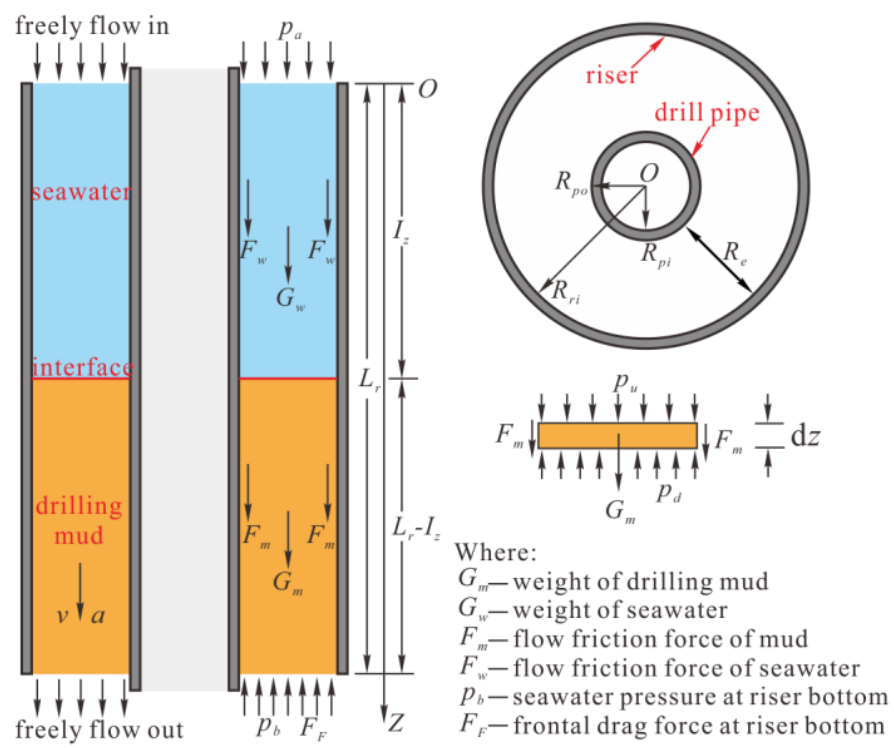

Fig. 1. Sketch of drilling mud discharging from a riser annulus

Some public parameters are defined in Table 1, and SI Units are applied for all parameters.

Table 1. Parameters definition for governing equations

\begin{tabular}{ll}
\hline Parameters & Symbol \\
\hline Vertical position begins with sea level & $z$ \\
Time starts after riser is disconnected & $t$ \\
Riser length(length of the annulus flow channel) & $L_{r}$ \\
Distance from sea level to top mud level(interface) & $I_{z}$ \\
Density of drilling mud \& seawater & $\rho_{m} \& \rho_{w}$ \\
Hydraulic diameter \& flow area of flow channel & $D_{e} \& A$ \\
Velocity \& acceleration of fluid discharge & $v \& a$ \\
Pressure in fluid column & $p$ \\
Friction coefficient of seawater \& drilling mud & $f_{w} \& f_{m}$ \\
Friction resistance per unit length of flow channel & $P_{f}$ \\
\hline
\end{tabular}

\section{Whole fluid column model}

The initial "slug column model" took mud column slug as the object, without considering the flow of refilled seawater(Yong et al., 1992). This model is concise in form and fast in program running, and can act as a good comparison for any other complicated simulation. But regrettably, relevant papers did not provide any detailed solving algorithms for reference.

According to the idea "slug column model", we present a modified model and name it as "whole fluid column(WFC)" model. First, loads and movement of the whole fluid column(drilling mud and refilled seawater) rather than the single mud section are discussed. Second, flow channel is the riser annulus instead of a single riser pipe. Third, a specific governing equation is proposed and a special algorithm by a cubic equation is developed. Fourth, annular friction force is calculated strictly.

For any time $t$ during discharge, applying Newton's Second Law to the whole fluid column, and a governing equation of WFC model can be established:

$$
\begin{aligned}
& \rho_{w} g A_{e} I_{z}+\rho_{m} g A_{e}\left(L_{r}-I_{z}\right)-2 f_{w} \frac{A e}{D_{e}} \rho_{w} v^{2} I_{z}-2 f_{m} \frac{A e}{D_{e}} \rho_{m} v^{2}\left(L_{r}-I_{z}\right) \\
& -\rho_{w} g A_{e} L_{r}-\frac{1}{2} \rho_{m} A v^{2}=\left[\rho_{w} A I_{z}+\rho_{m} A_{e}\left(L_{r}-I_{z}\right)\right] \cdot a
\end{aligned}
$$

Where, the 1 st and 2 nd items are the total weight of remainder mud and refilled seawater; the $3 \mathrm{rd}$ and 4 th items are the total friction force of two fluid columns; the 5th item is the force of seawater static pressure at riser bottom; the 6th item is the frontal drag force when falling column hits seawater at riser bottom; the 7th item is the multiplication of total column mass and falling acceleration. For riser annulus, $D_{e}$ is the difference between inner diameter of riser $D_{r i}$ and external diameter of drill pipe $D_{p o}$, i.e. $D_{e}=D_{r i}-D_{p o}$.

Setting equal time interval $\Delta t . a_{i}$ is the average acceleration in each time step (from $t_{i-1}$ to $t_{i}$ ) and $a_{0}=0 \mathrm{mx} \mathrm{s}^{2}\left(t_{0}=0 s\right.$ ); $v_{i}$ is the falling velocity at time $t_{i}$ and $I_{z i}$ is the interface location of the top mud level. At each time $t_{i}$ :

$v_{i}=v_{i-1}+a_{i} \cdot \Delta t, \quad I_{z i}=I_{z i-1}+v_{i-1} \cdot \Delta t+0.5 a_{i}(\Delta t)^{2}$

Then substitute Eq.(2) into Eq.(1) and rearrange it:

$B_{3} a_{i}^{3}+B_{2} a_{i}^{2}+B_{1} a_{i}+B_{0}=0$

Eq.(3) is a cubic equation of $a_{i}$ unknown at time $t_{i}$. Coefficients are:

$$
\begin{aligned}
B_{3} & =0.5 C_{1}(\Delta t)^{4} \\
B_{2} & =\left[C_{1}\left(I_{z i-1}+2 v_{i-1} \cdot \Delta t\right)+C_{2}+0.5 C_{3}\right] \cdot(\Delta t)^{2} \\
B_{1} & =\left[C_{1}\left(2 I_{z i-1}+2.5 v_{i-1} \cdot \Delta t\right)+2 C_{2}+C_{3}\right] \cdot v_{i-1} \cdot \Delta t \\
& +C_{3} I_{z i-1}+0.5 C_{4}(\Delta t)^{2}+C_{5} \\
B_{0} & =\left(C_{1} v_{i-1}^{2}+C_{4}\right)\left(I_{z i-1}+v_{i-1} \cdot \Delta t\right)+C_{2} v_{i-1}^{2}+C_{6}
\end{aligned}
$$

Where,

$$
C_{1}=\frac{2 A_{e}}{D_{e}}\left(f_{m} \rho_{m}-f_{w} \rho_{w}\right)
$$$$
C_{2}=-\left[\frac{2 A_{e}}{D_{e}} f_{m} \rho_{m} L_{r}+\frac{1}{2} \rho_{m} A\right]
$$

$C_{3}=\left(\rho_{m}-\rho_{w}\right) A$

$C_{4}=\left(\rho_{w}-\rho_{m}\right) g A$

$C_{5}=-\rho_{m} A L_{r}$ 
Friction coefficients $f_{w}$ and $f_{m}$ in Eq.(1) are computed by the method presented in Appendix A, in which different flow regimes are considered.

\section{CFD simulation}

Although WFC model is easy to realize, its velocity and acceleration are constant along the whole fluid column in each time step. In fact, after riser disconnection, a "fast-opening waterhammer" occurs immediately, and a pressure wave propagates upwards(Miller et al., 1998). WFC model can not simulate such an unsteady flow, i.e., $v(z, t), a(z, t), p(z, t), f_{w}(z, t)$ and $f_{n t}(z, t)$. In current section, we develop a CFD procedure to capture details of these flow parameters during mud release.

\subsection{Governing equation deduction}

First, considering gravity and flow friction, one dimensional Navier-Stokes Equation(momentum equation) in vertical direction is applied:

$\frac{\partial v}{\partial t}+\frac{1}{\rho} \frac{\partial p}{\partial z}+v \frac{\partial v}{\partial z}=g-\frac{P_{f}}{\rho A}$

Where, $P_{f}=2 A / D_{e} f \rho v^{2}$ is flow friction force per unit length of flow channel; $\rho$ is fluid density in flow channel; $f$ is friction coefficient which can be determined by the method in Appendix A.

And then, one-dimensional continuity equation is:

$\frac{\partial}{\partial z}(\rho v A)+\frac{\partial}{\partial t}(\rho A)=0$

Because compressibility is not obvious in common drilling mud and seawater, it is neglected in momentum equation Eq.(4) but considered in continuity equation Eq.(5).

Unfold Eq.(5) and divide each item by $\rho A_{\varepsilon}$ :

$\frac{v}{\rho} \frac{\partial \rho}{\partial z}+\frac{\partial v}{\partial z}+\frac{v}{A} \frac{\partial A}{\partial z}+\frac{1}{\rho} \frac{\partial \rho}{\partial t}+\frac{1}{A} \frac{\partial A_{e}}{\partial t}=0$

Considering the weak expansibility and compressibility of flow channel and fluid during pressure wave propagating, total derivative of flow area $A$ and fluid density $\rho$ are:

$\frac{\mathrm{d} A}{\mathrm{~d} t}=\frac{\partial A_{e}}{\partial t}+v \frac{\partial A_{e}}{\partial z}, \frac{\mathrm{d} \rho}{\mathrm{d} t}=\frac{\partial \rho}{\partial t}+v \frac{\partial \rho}{\partial z}$

Substitute Eq.(7) into Eq.(6) and we can get:

$\frac{1}{\rho} \frac{\mathrm{d} \rho}{\mathrm{d} t}+\frac{1}{A} \frac{\mathrm{d} A}{\mathrm{~d} t}+\frac{\partial v}{\partial z}=0$

Fluid compressibility coefficient is $\alpha=-\frac{\mathrm{d} V}{V} / \mathrm{d} p$; where, $V$ is fluid volume. And the total derivative of a fixed mass $m(m=\rho V)$ is:

$V d \rho+\rho \mathrm{d} V=\mathrm{d} m=0 \Rightarrow \frac{\mathrm{d} \rho}{\rho}=-\frac{\mathrm{d} V}{V}$, i.e., $\frac{1}{\rho} \frac{\mathrm{d} \rho}{\mathrm{d} t}=\alpha \frac{\mathrm{d} p}{\mathrm{~d} t}$

Expansibility coefficient of flow channel cross-section is:

$\beta=\frac{\mathrm{d} A_{e}}{A_{e}} / \mathrm{d} p$, i.e., $\frac{1}{A_{e}} \frac{\mathrm{d} A_{e}}{\mathrm{~d} t}=\beta \frac{\mathrm{d} p}{\mathrm{~d} t}$

And total derivative of fluid pressure is: $\frac{\mathrm{d} p}{\mathrm{~d} t}=\frac{\partial p}{\partial t}+v \frac{\partial p}{\partial z}$

Substitute Eqs.(9)-(11) into Eq.(8), and a new form of the continuity equation is built:

$v \frac{\partial p}{\partial z}+\frac{\partial p}{\partial t}+\frac{1}{\alpha+\beta} \frac{\partial v}{\partial z}=0$

Next, multiply each item of Eq.(4) by $\rho v$, then subtract Eq.(12) and we acquire a new equation:

$\rho v \frac{\partial v}{\partial t}-\frac{\partial p}{\partial t}+\rho v^{2} \frac{\partial v}{\partial z}-\frac{1}{\alpha+\beta} \frac{\partial v}{\partial z}=\rho v g-\frac{P_{f} v}{A}$

Set $v=1 / \sqrt{\rho(\alpha+\beta)}$ and $\kappa=2 f / D_{e}$, assemble Eq.(4) and Eq.(13) and then transform them into a matrix form. Finally, a governing equation for CFD method of fluid falling can be obtained:

$\mathbf{u}+\mathbf{F}_{z}=\mathbf{S}$

Where, $\quad \mathbf{Y}=\frac{\partial \mathbf{U}}{\partial t}, \quad \mathbf{F}_{z}=\frac{\partial \mathbf{F}}{\partial z}, \quad$ and $\quad \mathbf{U}=\left[\begin{array}{l}v \\ 0.5 \rho v^{2}-p\end{array}\right]$ $\mathbf{F}=\left[\begin{array}{l}v^{2} / 2+p / \rho \\ \rho v^{3} / 3-\rho v^{2} v\end{array}\right], \mathbf{S}=\left[\begin{array}{l}g-\kappa v^{2} \\ \rho v g-\kappa \rho v^{3}\end{array}\right]$

\subsection{Governing equation solution}

Eq.(14) is discretized by finite volume method:

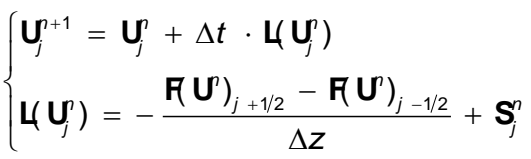

Where, $\mathbf{F} \mathbf{U}^{\mathbf{p}}$ ) is the numerical flux at time $t_{n}$.

In order to suppress oscillation along characteristic lines and enhance accuracy, flux splitting is needed before spatial discretization. That is:

$\mathbf{F}\left(\mathbf{U}^{\prime}\right)=\mathbf{F}\left(\mathbf{U}^{+}+\mathbf{F} \mathbf{U}^{\mathbf{2}}\right)^{-}$

Sterger-Warming flux splitting algorithm(Goldstein et al., 1993) is commonly used for Euler equations. Here, this algorithm is referenced to split the flux item of a fluid falling equation Eq.(14).

At first, the Jacobian matrix is:

$\mathbf{A}=\frac{\partial \mathbf{F} \mathbf{U})}{\partial \mathbf{U}}=\left[\begin{array}{cc}2 v & -1 / \rho \\ \rho\left(v^{2}-v^{2}\right) & 0\end{array}\right]$

And eigenvalues of matrix $\mathbf{A}$ are: $\lambda_{1}=v+v, \lambda_{2}=v-v$. For a fluid falling problem in riser, $v$ is the wave velocity in this annulus flow channel, and $v \square v$. Correspondingly, eigenvalues matrix $\Lambda$ is split as follow:

$\Lambda=\Lambda^{+}+\Lambda^{-} \Rightarrow\left[\begin{array}{cc}\lambda_{1} & 0 \\ 0 & \lambda_{2}\end{array}\right]=\left[\begin{array}{cc}v+v & 0 \\ 0 & 0\end{array}\right]+\left[\begin{array}{cc}0 & 0 \\ 0 & v-v\end{array}\right]$

Left eigenvectors matrix $\mathbf{L}$ and right eigenvectors matrix $\mathbf{R}$ are:

$\mathbf{L}=\left[\begin{array}{ll}(v+v) / 2 v & -1 / 2 \rho v \\ (v-v) / 2 v & 1 / 2 \rho v\end{array}\right], \mathbf{R}=\left[\begin{array}{cc}1 & 1 \\ \rho(v-v) & \rho(v+v)\end{array}\right]$

Where, $\mathbf{A}=\mathbf{R} \Lambda \mathbf{L}$.

Finally, numerical flux after splitting can be obtained by:

$\mathbf{F} \mathbf{U}^{ \pm}=\mathbf{A}^{ \pm} \mathbf{U}=\mathbf{R} \Lambda^{ \pm} \mathbf{U}$ 
It is important to discretize the convective term $\mathbf{F}_{z}$ of Eq.(14) by a high-precision scheme. That is because: first, variations of flow parameters are drastic in the initial period of mud falling; and second, discontinuity exists(e.g. density gradient) at each interface of drilling mud and refilled seawater. The fifth-order weighted essentially nonoscillatory(WENO) scheme(Shu, 1988; Osher, 1988; Liu et al., 1994) is a proper scheme that can ensure a smooth and oscillation free solution for large gradients while at the same time maintain a high order of accuracy. Hence, we utilize this scheme for convective term discretization.

Specifically, after flux splitting at every position node, five interpolation values are chosen to determine $\left.\mathbf{F} \mathbf{U}^{\mathbf{r}}\right)_{j+1 / 2}^{ \pm}$, i.e., set:

$\left\{\begin{array}{ll}\mathbf{F} \mathbf{U}_{j+1 / 2}^{+}: & \eta_{k}^{+}=\mathbf{F}_{j+k-3 ;} ; \\ \mathbf{F} \mathbf{U}_{j+1 / 2}^{-}: & \eta_{k}^{-}=\mathbf{F}_{j-k+4} ;\end{array} \quad(k=1,2, \cdots, 5)\right.$.

Then, replace $q_{k}$ of Eqs.(B.1, B.2) in Appendix B by $\eta_{k}^{+}$or $\eta_{k}^{-}$ respectively. And via Eqs.(B.1-B.4), the final item $q_{j}^{\text {væDO }}$ is the result of $\left.\mathbf{R} \mathbf{U}^{\mathbf{r}}\right)_{j+1 / 2}^{+}$or $\left.\mathbf{F} \mathbf{U}^{\mathbf{1}}\right)_{j+1 / 2}^{-}$. Next:

$$
\mathbf{F}\left(\mathbf{U}^{\prime}\right)_{j+1 / 2}=\mathbf{F}\left(\mathbf{U}^{+}\right)_{j+1 / 2}+\mathbf{F}\left(\mathbf{U}^{-}\right)_{j+1 / 2}^{-}
$$

$\left.\mathbf{F} \mathbf{U}^{\mathbf{r}}\right)_{j-1 / 2}$ can be got by a similar way at a prior spatial step.

Time discretization of Eq.(15) is realized by the third order total variation diminishing(TVD) Runge-Kutta scheme(Shu, 1988). Eq.(C.1) in Appendix C shows the detail.

\subsection{Interface tracking by Level Set method}

During mud discharge, the space emptied out in the upper riser is instantly replaced by seawater through refill valves. Interface of top mud level is a basic factor to compute other parameters in the two column sections. In this paper, Level Set method(Shu, 1988; Adalsteinsson and Sethian, 1999; Chen et al., 1997) is utilized to track the moving interface for the first time. This method adopts a smooth signed distance function $\varphi(\vec{z}, t)$ and its zero level set $\Gamma(t)$ represents the interface location:

$\varphi(z, t)= \begin{cases}-d(z, \Gamma(t)), & z \in \Omega_{i} ; \\ 0, & z=\Gamma(t) ; \\ d(z, \Gamma(t)), & z \in \Omega_{2} .\end{cases}$

Where, $\Omega_{1}$ and $\Omega_{2}$ represent the section of refilled seawater and the section of residual mud, respectively; $d(z, \Gamma(t))$ is the distance between position $\vec{z}$ and interface $\Gamma(t)$.

For mud falling in this annulus flow channel, interface $\Gamma(t)$ is moved by a velocity field $\vec{\nabla}$. Hence, an one-dimensional convection equation in vertical direction, i.e., Level Set function, is appropriate to describe the changing of $\varphi(\vec{z}, t)$ :

$\varphi_{t}+v \cdot \varphi_{z}=0$

Spatial item $\left(\varphi_{z}\right)_{j}$ at each position $z_{j}$ is obtained by the fifth order WENO scheme and based on the sign of $v_{j}$. If $v_{j} \geq 0$, $\left(\varphi_{z}\right)_{j}=\left(\varphi_{z}\right)_{j}^{-}$; if $v_{j}<0,\left(\varphi_{z}\right)_{j}=\left(\varphi_{z}\right)_{j}^{+} \cdot\left(\varphi_{z}\right)_{j}^{ \pm}$are determined as follows.
At first, for each spatial step $z_{j}$, set five interpolation values:

$\left\{\begin{array}{ll}\left(\varphi_{z}\right)_{j}^{+}: & \psi_{k}^{+}=\left(\varphi_{j-k+4}-\varphi_{j-k+3}\right) / \Delta z ; \\ \left(\varphi_{z}\right)_{j}^{-}: & \psi_{k}^{-}=\left(\varphi_{j+k-3}-\varphi_{j+k-4}\right) / \Delta z ;\end{array} \quad(k=1,2, \cdots, 5)\right.$.

And then, replace $q_{k}$ of Eqs.(B.1, B.2) in Appendix B with $\psi_{k}^{+}$or $\psi_{k}^{-}$respectively. And via Eqs.(B.1-B.4), the item $q_{j}^{\text {VEDO }}$ in Eq.(B.4) is the result of $\left(\varphi_{z}\right)_{j}^{+}$or $\left(\varphi_{z}\right)_{j}^{-}$.

Next, time discretization of Eq.(21) is realized by the Runge-Kutta scheme in Appendix C:

$\varphi_{j}^{n+1}=\varphi_{j}^{n}+\Delta t \cdot\left(-v_{j} \cdot\left(\varphi_{z}\right)_{j}^{n}\right)$

When the interface moves, the Level Set function looses its signed distance property. In order to maintain this property and ensure mass conservation, the Level Set function needs to re-initialize after each time step. Here, we adopt the modified Godunov method(Chen et al., 1997; Fedkiw, 1999). The re-initialization equation is:

$\varphi_{t}+S\left(\varphi_{0}\right)\left(\left|\varphi_{z}\right|-1\right)=0$

Where, the smoothed signed function is $S\left(\varphi_{0}\right)=\varphi_{0} / \sqrt{\varphi_{0}^{2}+\Delta Z^{2}}$.

And set $S=\frac{S\left(\varphi_{0}\right)\left(\left|\varphi_{z}^{+}\right|-\left|\varphi_{z}^{-}\right|\right)}{\varphi_{z}^{+}-\varphi_{z}^{-}}$. Thus, at each spatial step, $\varphi_{z}$ is determined by Eq.(25):

$$
\varphi_{z}=\left\{\begin{array}{lll}
\varphi_{z}^{-}, & \mathbb{S}\left(\varphi_{0}\right) \varphi_{z}^{+} \geq 0, & \mathbb{S}\left(\varphi_{0}\right) \varphi_{z}^{-} \geq 0, \\
\varphi_{z}^{+}, & \mathbb{S}\left(\varphi_{0}\right) \varphi_{z}^{+} \leq 0, & \mathbb{S}\left(\varphi_{0}\right) \varphi_{z}^{-} \leq 0, \\
0, & \mathbb{S}\left(\varphi_{0}\right) \varphi_{z}^{+}>0, & \mathbb{S}\left(\varphi_{0}\right) \varphi_{z}^{-}<0, \\
\varphi_{z}^{-}, & \mathbb{S}\left(\varphi_{0}\right) \varphi_{z}^{+}<0, & \mathbb{S}\left(\varphi_{0}\right) \varphi_{z}^{-}>0, s>0, \\
\varphi_{z}^{+}, & \mathbb{S}\left(\varphi_{0}\right) \varphi_{z}^{+}<0, & \mathbb{S}\left(\varphi_{0}\right) \varphi_{z}^{-}>0, s \leq 0 .
\end{array}\right.
$$

Where, $\left(\varphi_{z}\right)_{j}^{+}$and $\left(\varphi_{z}\right)_{j}^{-}$can be computed via the same procedure mentioned above. Similarly, time discretization of Eq.(24) is implemented by Eq.(C.1).

In order to acquire a stable solution of $\varphi(\vec{z}, t)$, iteration is necessary during this re-initialization. The corresponding termination condition is: $\sum_{j}\left|\varphi_{j}^{n+1}-\varphi_{j}^{n}\right|<\varepsilon$; in this paper, $\varepsilon=0.01 \Delta z$. After 3-4 iterations, a desirable re-initialized $\phi(\vec{z}, t)$ can be achieved.

\subsection{Boundary and interface treatment}

In current discussion, the top boundary is sea level which has a constant zero pressure, and seawater flows freely into the riser. While for the bottom boundary, the frontal drag force at riser bottom is considered, i.e., the pressure at riser bottom is the sum of static seawater pressure and the equivalent pressure of frontal drag force: $\rho_{w} g L_{r}+0.5 \rho_{m} v_{b}^{2}$.

When implementing spatial discretization for Eqs. $(18,22)$ by WENO scheme, six grid points are needed at each spatial step. Ghost Method(Fedkiw et al., 1999) is referenced for extending those grids near interface, while virtual staggered grids and interpolation technique are applied for boundary grids(Fig. 2). 

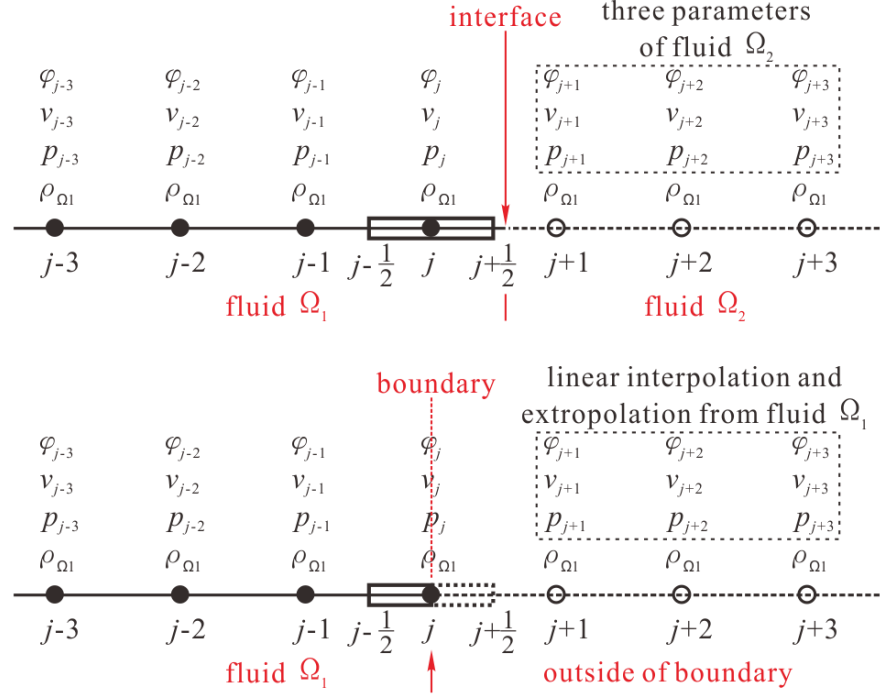

Fig. 2. Grids treatment around interface and boundary

\subsection{CFD simulation steps}

In order to realize CFD simulation, we develop a special MATLAB program. Actually, MATLAB is a very useful mathematical tool and can provide efficient simulation in many fields of science. For example, Valipour et al.(2012, 2013) successfully forecasted the monthly inflow of Dez dam reservoir by the autoregressive artificial neural network models, and they realized their complicated algorithms in MATLAB software environment. In this paper, CFD method is a time-domain process and several parameters are needed to compute at each time step. Therefore, we take the advantages of MATLAB software to carry out matrixes operations. Main steps of CFD method are listed as follows:

(1) Initialize $v(z, 0), p(z, 0), f(z, 0)$ and $\varphi(z, 0)$.

(2) Compute time step length $\mathrm{d} t, \mathrm{~d} t=\mathrm{CF} \cdot \mathrm{d} z /|\lambda|_{\max }$.

(3) Calculate $\varphi(z, t)$ and interface $\Gamma(t)$, determine $\rho$ at each column section, and then re-initialize $\varphi(z, t)$.

(4) Split flux $\left.\mathbf{F} \mathbf{U}^{ \pm}\right)^{ \pm}$by Steger-Warming method, discretize spatial item by WENO scheme, calculate $f(z, t)$ and source item $\mathbf{S}$, and apply boundary conditions.

(5) Discretize temporal item and determine $v(z, t), p(z, t)$ and $a(z, t)$.

(6) Repeat steps (2)-(5).

(7) Calculate total column mass, column weight, weightlessness and friction force.

\section{Methods verification and case discussion}

\subsection{Methods verification}

Although contrastive analysis between numerical simulation and field measurement are usually the most effective way for method verification, it is hard to do field measurement or experimental simulation of the unsteady flow of mud discharge, and there is nearly no reliable metrical data and experimental data in any published references of this problem. Therefore, comparison with some published simulation results is an alternative but not perfect way. However, simulation data of mud discharge in previous papers are also inadequate or incomplete. In current section, results of the two methods are compared with two other papers.

At first, by adopting the same data from a master thesis of Grønevik(2013), mud discharge is simulated by both WFC method and CFD method. This case is an pipe flow with no drill pipe in the riser; riser length is $500 \mathrm{~m}$, mud density is $1600 \mathrm{~kg} \mathrm{~m}^{3}$, and inner diameter of the riser is $0.48895 \mathrm{~m}$. The compared results(Fig.3) show that discharge velocity, length of mud column and friction force on the riser during the whole releasing time coincide well with each other between WFC method and CFD method, and are also close to the results of Grønevik.

Then, the results of the current two methods are compared with the results by $\mathrm{Li}$ et al.(2012). Li's results were calculated by the 2HRECOIL software. In current case(the main case), riser length, mud density and rheological parameters are the same as the data by Li's paper. Table 2 lists nine essential parameters needed by WFC method and CFD method.

Table 2. Given data of the main case

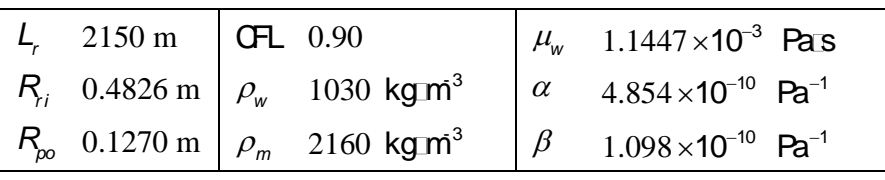

Herschel-Bulkley rheological model $\left(\tau=\tau_{0}+K \gamma^{n}\right)$ is applied in this case. Where, yield stress $\tau_{0}$ is $3 \mathrm{~Pa}$, shear rate power $n$ is 0.6 , and fluid consistency $K$ is 0.16 Pas.

Fig. 4 shows three contrastive curves by our methods and Li's results. The maximal discharge velocity (WFC: $14.27 \mathrm{~m} / \mathrm{s}$, CFD $14.61 \mathrm{~m} / \mathrm{s}$ ) and the maximal friction force on riser(WFC: $3019.0 \mathrm{kN}$, CFD: $3091.8 \mathrm{kN})$ are almost equal to Li's results(14.39 $\mathrm{m} / \mathrm{s}$ and $3078.4 \mathrm{kN}$ respectively).

However, large deviation exists between our methods and Li's model. The possible reasons are: First, Li's model took the flow state of the bottom column section(near LMRP) as the state of the whole column, but not considered the response time difference between bottom and top; while, WFC method calculates the average velocity and friction force of the whole fluid column, and CFD method computes velocity at each depth and friction force of the whole column. Second, neither the shape(pipe flow or annulus flow) nor the size(riser diameter) of the flow channel in Li's case were introduced in his paper, and friction coefficients calculation in this paper is different from Li's paper.

In fact, both flow channel shape and friction coefficients have great influence on final simulation results. In order to illustrate this point, Fig. 5 displays three different situations:

$\mathrm{L}_{1}$ : pipe flow(p-f, with no drill pipe in riser), with variable friction coefficients $\left(v-f_{w}-f_{m}\right)$;

$\mathrm{L}_{2}$ : annulus flow(a-f, riser and drill pipe), with constant friction coefficients $\left(\mathrm{c}-f_{w}-f_{m}, f_{w}=f_{m}=0.002\right)$;

$\mathrm{L}_{3}$ : annulus flow(a-f), with variable friction coefficients $\left(v-f_{w}-f_{m}\right)$.

In annulus flow $\left(\mathrm{L}_{3}\right)$, part of the friction force is provided by the outer wall of drill pipe, so the friction force on riser is lower than pipe 
flow $\left(\mathrm{L}_{1}\right)$. However, annulus flow is closer to the real disconnection situation than pipe flow. Also, because of the large variation of velocity, Reynolds number changes in a very wide range(from $10^{\circ}$ to $10^{6}$ ), and correspondingly, friction coefficients are not two constant values but change with velocity variation. Therefore, annulus flow model with variable friction coefficients $\left(\mathrm{L}_{3}\right)$ provides more accurate simulations. Given that precise friction coefficient is an important prerequisite for mud discharge simulation, this paper calculates friction coefficients carefully according to different fluid type, flow velocity, flow regime and rational rheological model(Appendix A, Fig. 6).

\subsection{Case discussion}

Based on the given data(Table 2, annulus flow) and strict computation of friction coefficients(Fig. 6), mud discharge is simulated by both WFC method and CFD method. Figs. 7-15 reveal some important details, and all contrastive curves of entire trends(Figs. 7-10,12) coincide well with each other between the two methods(WFC, CFD). This can indirectly prove the reliability of the two methods.

The whole mud column is replaced by seawater gradually and the total discharge time is $195.83 \mathrm{~s}$ (by CFD). For the overall trends, discharge acceleration(Fig. 8) reaches its maximal average value(5.04 $\mathrm{m} / \mathrm{s}^{2}$ by WFC) at the beginning of mud falling, and then decreases sharply to zero at $10.5 \mathrm{~s}$ and fluctuates around zero in the whole later time. Correspondingly, discharge velocity(Fig. 10) reaches its maximum(14.61 $\mathrm{m} / \mathrm{s}$ by CFD) at $10.5 \mathrm{~s}$, and then decreases slowly during the whole discharge process.

Because of the biggish acceleration in the first $10.5 \mathrm{~s}$ (Fig. 8), fluid column keeps in weightlessness state (Fig. 9) with a maximal weight loss value $4037.4 \mathrm{kN}(7756.4 \mathrm{kN}-3718.9 \mathrm{kN})$ at $8.7 \mathrm{~s}$, which is nearly half of the whole column weight. Such weightlessness reduces the weight of whole riser system and may increase the recoil acceleration by the redundant top tension. If velocity of riser recoil is out of control and riser tensioners move beyond their strokes, the riser top may crash floating platform and result in undesirable accident.

Similarly, friction force(Fig.9) increases quickly to its maximal value $(3905.4 \mathrm{kN})$ in the first $8.7 \mathrm{~s}$, while decreases smoothly in the rest discharge duration. Actually, friction force on inner riser wall is a crucial concern of riser recoil. We use a multiplier(ratio of wetted perimeter, i.e., $\left.D_{r i} /\left(D_{r i}+D_{p o}\right)\right)$ to obtain it from the total friction force of annulus. And its maximum(3091.8 kN) is huge, nearly half of total column weight. Such a large value would effectively cushion riser recoil upwards, nevertheless, would threaten the safe clearance between riser bottom and subsea equipment after disconnection.

In Fig. 12, fluid pressure at each depth attenuates sharply in the first $5 \mathrm{~s}$, and then maintains a relatively constant pressure which is equal to the seawater pressure at the same depth outside riser. Moreover, a smooth fluctuation exists at each depth and this indicates the moment that the interface reaches, e.g., $500 \mathrm{~m}$ at $37.4 \mathrm{~s}, 1000 \mathrm{~m}$ at $74.5 \mathrm{~s}, 1500 \mathrm{~m}$ at $115.1 \mathrm{~s}$ and $2000 \mathrm{~m}$ at $167.9 \mathrm{~s}$.

From the contrastive curves we can see that WFC method can only offer average value of each flow parameter at whole depth, while
CFD method can simulate several sensitive fluctuations very well at each fluid layer. In Fig. 8, after sharply dropping, acceleration fluctuates up and down around zero, and maintains a small minus average value at each time $\operatorname{step}\left(\right.$ minimum value is $-0.147 \mathrm{~m} / \mathrm{s}^{2}$ ). In addition, amplitude of acceleration fluctuation becomes larger when interface arrives. Meanwhile, velocity continuously oscillates during the whole mud falling duration. Actually, each small oscillation of acceleration and velocity corresponds to one trip of pressure wave propagation or reflection along the riser annulus. For a $2150 \mathrm{~m}$ annulus filling with static fluid, the maximal propagation period of pressure wave is $2.438 \mathrm{~s}$ when the whole annulus is single mud, while minimum time for one trip is $1.684 \mathrm{~s}$ when the whole annulus is static seawater.

Figs. 11 and 14 show the drastic variation of velocity along column depths in the first few seconds. Because of inertia force and viscous force, the upper column layer keeps static before the first pressure wave reaches, and the top of mud column(depth $0 \mathrm{~m}$ ) starts response at $2.29 \mathrm{~s}$. Because the static layers at the upper column can't provide enough column pressure to each lower layer, lower layers have velocity dropping after initial acceleration. And then, when all fluid layers begin falling, velocity distribution at every depth comes to uniform gradually, and the whole column reaches its maximal velocity value at $10.5 \mathrm{~s}$.

Figs. 13 and 15 exhibit the details of pressure attenuation in the first few seconds. Pressure dropping begins from riser bottom to riser top gradually, and after $2.29 \mathrm{~s}$ the top layer(depth $0 \mathrm{~m}$ ) begins response. At each depth, pressure drops sharply to a low pressure at first, and then recovers to the value equivalent to the outside static seawater pressure at the same depth. The "low pressure" is far below the static seawater outside riser from $0 \mathrm{~m}$ to $1500 \mathrm{~m}$ and lasts $1-3 \mathrm{~s}$ according to depth. Particularly, pressure drops to zero from $0 \mathrm{~m}$ to $925 \mathrm{~m}$. It is worth emphasizing that negative pressure in Miller's paper(Miller et al., 1998) is impossible; once the fluid pressure drops to zero during mud falling, fluid vaporization would generate and thus vacuum can not maintain. Although such pressure attenuation is fleeting in time, the depth range of riser affected by pressure drop is very long. More seriously, if the pressure in riser drops too low to the static seawater pressure outside riser, riser body would collapse by large pressure difference. In current case(Figs. 13 and 15), pressure differences are: $5.41 \mathrm{MPa}$ at $1500 \mathrm{~m}, 9.18 \mathrm{MPa}$ at $1000 \mathrm{~m}$ and 5.05 $\mathrm{MPa}$ at $500 \mathrm{~m}$ respectively, while the maximal pressure difference is $9.41 \mathrm{MPa}$ at $950 \mathrm{~m}$ and $1.603 \mathrm{~s}$.

\subsection{Potential applications of the methods and results}

In our MATLAB practice, WFC method is very concise and operates very fast compared with the complicated CFD method(running time is $0.378 \mathrm{~s}$ vs $144.266 \mathrm{~s}$ when element length is $25 \mathrm{~m}$ ). WFC method can quickly provide several reliable parameters such as total column mass and weight, friction force, velocity and pressure. Hence, it is convenient for a structural analysis of a riser(static or dynamic) in hanging state after emergency disconnection. However, because WFC method applies constant velocity and constant acceleration along the whole fluid column at each time step, it is impossible to simulate every detailed fluctuation 
at each fluid layer for riser recoil analysis.

Conversely, CFD method is sensitive enough to capture every small flow fluctuation in the first few seconds, which is the main period of riser recoil. Therefore, it is advisable to integrate this procedure into a structural model and provide more accurate hydraulic results for riser recoil response analysis. Specifically, velocity variation determines drag loads along inner riser wall, fluid pressure affects distribution of effective tension of riser, and fluid column mass is a prerequisite to compute exact system weight.

In terms of the results, much attention should be paid on the opposite influence of fluid weightlessness and friction force(Fig. 9) during mud discharge: fluid weightlessness accelerates riser recoil, and may induce the riser top impacting platform in a sudden time; friction force restrains riser leaving instantly from BOP after disconnection, and may cause the riser bottom striking subsea equipment. Therefore, it is necessary to implement a comprehensive analysis of the two contrary effects in a structural model of riser recoil, and accordingly, design rational riser top tension and recoil control measures. In addition, since the drastic pressure drop in riser in the first few seconds after an emergency disconnection, excess strength of resisting external pressure should be considered during riser selection, and setting several refill valves along different depth of riser may be another insurance measure.

\subsection{Uncertainties and sensitivity analysis}

As has been discussed above, mud discharge is a kind of complicated unsteady flow. Structure of flow channel and properties of drilling mud are two main aspects that affecting the release process:

(1) flow channel: length, size, shape, degree of eccentricity, roughness of pipe wall, connector of drill pipe, and opening manner of riser end.

(2) drilling mud: density, viscosity, reheology model, and initial velocity.

In order to acquire more explicit cognition of this problem, contrastive analysis is needed to evaluate the specific sensitivity of these parameters in further research. Especially, eccentricity, roughness and opening manner(including distribution of refill valves) are all significant factors in real situation, but it is hard to determine them in any simulation. In current paper, they are all been simplified or neglected, and this may be the main error sources in our calculations.

In addition, after drilling riser disconnected, riser would recoil upwards immediately during mud releasing. Thus, outer boundary of annulus flow channel has varying velocities at each time. In this paper, vertical shift of riser is ignored. However, this can be considered by coupling our methods to a structural model of riser recoil.

Another uncertainty is that fluid vaporization would generate if fluid pressure drops to zero during mud falling. Once vaporization happens, gas will greatly change the property of both drilling mud and refilled seawater. It is very difficult to determine the degree of vaporization during that fleeting time and how mud influence on possible multiphase flow. Therefore, serious experiment is needed in next research of riser recoil problem.

\section{Conclusions}

This paper develops two fresh mud discharge simulation procedures: WFC method and CFD method, including governing equations establishing and specific solve procedures. WFC method is concise in form and fast to operate; while CFD method, which uses the WENO scheme, Runge-Kutta scheme and Level Set function, is sensitive enough to capture every small fluctuation of flow parameters, especially variations of velocity and pressure at the first few seconds. Two methods can be integrated into a riser structural model for recoil response prediction or hanging riser dynamic analysis. Particularly, in both methods, friction coefficient is a decisive parameter and should be calculated according to flow velocity, flow regime and rheological model.

After riser bottom opened, velocity and friction force sharply increase in the first few seconds, and then decrease slowly during the rest whole discharge process. Meanwhile, pressure in riser at each depth drops rapidly in the initial period, and negative pressure difference generates along riser and lasts 1-3 $\mathrm{s}$ when the first pressure wave reaches at each depth, which may result in riser collapse. Moreover, in the initial period of riser disconnection, large weightlessness generated by falling acceleration may accelerate riser recoil, while friction force may threaten the safe clearance between riser bottom and subsea equipment. Therefore, special considerations are needed on riser collapse resistance, top tension design and riser recoil control. Since mud discharge during riser recoil is a kind of complicated unsteady flow, further efforts should be made to evaluate the specific influence of eccentricity, roughness and distribution of refill valves. Also, field measurement and experimental simulation are urgently necessary to verify the numerical results.

\section{Acknowledgements}

This paper is funded by two projects of the "National Science Foundation of China". The first project is "Research on mechanical property of drilling riser-conductor casing and stability of subsea wellhead in deepwater", and the accession number is 51574261. The second project is "Analysis of vibration characteristics of production pipes induced by high speed fluid in high pressure gas well", and the accession number is 51274219 .

\section{References}

Arid Grønevik, 2013. Simulation of drilling riser disconnection-recoil analysis. Master thesis of Norwegian University of Science and Technology, 38-40.

Chi-Wang Shu, 1988. Efficient implementation of essentially nonoscillatory shock-capturing schemes. Journal of Computational Physics, 77(2): 439-471. DOI: 10.1016/0021-9991(88)90177-5.

D. Adalsteinsson and J. A. Sethian, 1999. The fast construction of extension velocities in Level Set methods. Journal of Computational Physics, 148(1): 2-22. DOI: 10.1006/jcph.1998.6090.

D. Goldstein, R. Handler and L. Sirovich, 1993. Modeling a no-slip flow boundary with an external force field. Journal of Computational Physics, 105(2): 354-366. DOI: 10.1006/jcph.1993.1081.

Dominique Guillot, 1990. 4 Rheology of Well Cementing Slurries, Well Cementing. Developments in Petroleum Science, 28: 4-1-437. DOI: 10.1016/S0376-7361(09)70302-4.

Donogh W. Lang, James Real and Michael Lane, 2009. Recent 
developments in drilling riser disconnect and recoil analysis for deepwater applications. OMAE2009-79427. DOI: 10.1115/OMAE2009-79427.

Guttorm Grytoyr, Partha Sharma and Srinivas Vishnubotla, 2011. Marine drilling riser disconnect and recoil analysis. AADE-11NTCE-80.

Haobo Zhou, Honghai Fan, Yinghu Zhai and Qi Peng, 2014. A new utility calculation model for axial flow of non-Newtonian fluid in concentric annuli. Canadian Journal of Chemical Engineering, 92(5): 945-952. DOI: 10.1002/cjce.21963.

Honghai Fan, Haobo Zhou, Guo Wang, Qi Peng and Ying Wang, 2014. Utility hydraulic calculation model of Herschel-Bulkley rheological model for MPD hydraulics. SPE-171443-MS. DOI: 10.2118/171443-MS.

Jack E. Miller, Matthew J. Stahl and Christopher J. Matice, 1998. Riser collapse pressures resulting from release of deepwater mud columns. OTC 8853. DOI: 10.4043/8853-MS.

Mohammad Valipour, Mohammad Ebrahim Banihabib, Seyyed Mahmood Reza Behbahani, 2013. Comparison of the ARMA, ARIMA, and the autoregressive artificial neural network models in forecasting the monthly inflow of Dez dam reservoir. Journal of Hydrology, 476: 433-441. DOI: 10.1016/j.jhydrol.2012.11.017.

M. Valipour, M. E. Banihabib, S. M. R. Behbahani, 2012. Monthly inflow forecasting using autoregressive artificial neural network. Journal of Applied Sciences, 12(20): 2139-2147. DOI: 10.3923/jas.2012.2139.2147.

R. D. Yong, C. J. Hock, Geir Karlsen and J. E. Miller, 1992. Analysis and design of anti-recoil system for emergency disconnect of a deepwater riser: case study. OTC 6891. DOI: 10.4043/6891-MS.

Ronald P Fedkiw, Tariq Aslam, Barry Merriman and Stanley Osher, 1999. A non-oscillatory Eulerian approach to interfaces in multimaterial flows (the Ghost Fluid method). Journal of Computational Physics, 152(2): 457-492. DOI: 10.1006/jcph.1999.6236.

R. R. Paula Jr and D. R. Fonseca, 2013. Emergency disconnection guidelines. OTC 24418. DOI: 10.4043/24418-MS.

Saad El-Din M. Desouky and Nour A. El-Emam, 1990. A generalized pipeline design correlation for pseudoplastic fluids. Journal of Canadian Petroleum Technology, 29(5): 48-54. DOI: 10.2118/90-05-02.

S. Chen, B. Merriman, S. Osher and P. Smereka, 1997. A simple Level Set method for solving Stefan problems. Journal of Computational Physics, 135(1): 8-29. DOI: 10.1006/jcph.1997.5721.

Songcheng Li, Mike Campbell and Hugh Howells, 2012. Effect of mud shedding on riser anti-recoil control at emergency disconnect. OMAE2012-83764. DOI: 10.1115/OMAE201283764.

Stanley Osher, 1988. Fronts propagating with curvature-dependent speed: algorithms based on Hamilton-Jacobi formulations. Journal of Computational Physics, 79(1): 12-49. DOI: 10.1016/0021-9991(88)90002-2.

Xu-Dong Liu, Stanley Osher and Tony Chan, 1994. Weighted essentially non-oscillatory schemes. Journal of Computational Physics, 115(1): 200-212. DOI: 10.1006/jcph.1994.1187.

\section{Appendixes}

\section{A. Flow friction coefficient calculation}

Flow friction coefficient $f$ (or $f_{w}, f_{m}$ ) is an important parameter in governing equation Eq.(1) and Eq.(14), and it changes with position and time at each step. The following provides the specific formulas for an annular unsteady flow of mud discharge.

First, roughness of all pipe walls is neglected. Then, friction coefficient is computed based on different flow regimes. Laminar flow(LMF) and transition flow(TSF) transitorily exist in the initial period of mud falling. After accelerating, the flow turns into turbulent flow(TBF), and TBF lasts until the end of mud discharge.

For refilled seawater (Newtonian fluid), set Pe as Reynolds number, in which equivalent pipe diameter is used for annulus flow.

$$
f_{w}=\left\{\begin{array}{llc}
16 / \mathrm{Pe}, & \mathrm{LMF}, & \mathrm{Pe} \leq 232 \mathrm{Q} \\
6.25 \times 10^{-4} \mathrm{Pe}^{1 / 3}, & \mathrm{TSF}, & 2320 \leq \mathrm{Pe} \leq 4000 \\
0.0791 / \mathrm{Pe}^{1 / 4}, & \mathrm{TBF}, & 4000<\mathrm{Pe} \leq 10^{5} ; \\
0.0008+\frac{0.05525}{\mathrm{Pe}^{0.237}}, & \mathrm{TBF}, & \mathrm{Pe}>10^{5} .
\end{array}\right.
$$

For drilling mud (non-Newtonian fluid), we apply a distinguishing criterion of flow regime from Guillot(1990):

$\begin{cases}0<\mathrm{Pe}_{g} \leq \mathrm{Pe}_{L T}, & \mathrm{LMF} ; \\ \mathrm{Pe}_{L T}<\mathrm{Pe}_{g} \leq \mathrm{Pe}_{\pi}, & \mathrm{TSF} ; \quad \text { Wher e, }\left\{\begin{array}{l}\mathrm{Pe}_{L T}=3470-1370 n^{\prime} \\ \mathrm{Pe}_{g}>\mathrm{Pe}_{\pi},\end{array} \text { TBF. }\right.\end{cases}$

And friction coefficient $f_{m}$ is:

$f_{m}= \begin{cases}16 / \mathrm{Pe}_{g}, & \mathrm{LMF}, \\ \text { I nt er pol at } \mathrm{i} \text { on bet ween LMF and TBF, } & \text { TSF; } \\ f_{m}=0.125 n^{\prime \sqrt{n^{\prime}}}\left[0.0112+\mathrm{Pe}_{g}^{-0.3185}\right], & \text { TBF. }\end{cases}$

Where, $\mathrm{Pe}_{g}$ is generalized Reynolds number; $n^{\prime}$ is generalized rheological behavior index; $f_{m}$ of TBF in Eq.(A.3) comes from Desouky and El-Emam(1990).

$\mathrm{Pe}, \mathrm{Pe}_{g}$ and $n^{\prime}$ at annulus should be calculated according to a proper rheological model. Commonly, Herschel-Bulkley model and Four Parameters model are more precise for drilling mud than Bingham model or Power Law model. Some previous rheology references have offered applicable answers, e.g., Zhou et al.(2014) and Fan et al.(2014)

\section{B. The fifth-order WENO scheme}

For five interpolation values $q_{k}(k=1,2, \cdots, 5)$ in each spatial cell $Z_{j}$, the general WENO reconstruction process are listed here:

First, three interpolation polynomials are:

$\left\{\begin{array}{l}q^{1)}=q_{1} / 3-7 q_{2} / 6+11 q_{3} / 6 \\ q^{(2)}=-q_{2} / 6+5 q_{3} / 6+q_{4} / 3 \\ q^{(3)}=q_{3} / 3+5 q_{4} / 6-q_{5} / 6\end{array}\right.$

Second, three smoothness indicators are: 
$\left\{\begin{array}{l}I S_{1}=\frac{13}{12}\left(q_{1}-2 q_{2}+q_{3}\right)^{2}+\frac{1}{4}\left(q_{1}-4 q_{2}+3 q_{3}\right)^{2} \\ I S_{2}=\frac{13}{12}\left(q_{2}-2 q_{3}+q_{4}\right)^{2}+\frac{1}{4}\left(q_{2}-q_{4}\right)^{2} \\ I S_{3}=\frac{13}{12}\left(q_{3}-2 q_{4}+q_{5}\right)^{2}+\frac{1}{4}\left(3 q_{3}-4 q_{4}+q_{5}\right)^{2}\end{array}\right.$

Third, three weights $w_{k}$ are:

$\left\{\begin{array}{l}\xi_{1}=0.1, \quad \xi_{2}=0.6, \quad \xi_{3}=0.3 \\ \sigma_{k}=\xi_{k} /\left(\varepsilon+/ S_{k}\right)^{2} ; \\ w_{k}=\sigma_{k} / \sum_{k=1}^{3} \sigma_{k} ; \quad(k=1,2,3) .\end{array}\right.$

Where, $\varepsilon=10^{-5}$ is a small positive number to avoid zero denominator if $I S_{k}$ equals zero.

Finally, the combined polynomial for cell $Z_{j}$ after reconstruction is:

$q_{j}^{W \boxplus D O}=\sum_{k=1}^{3} w_{k} \cdot f^{(k)}$
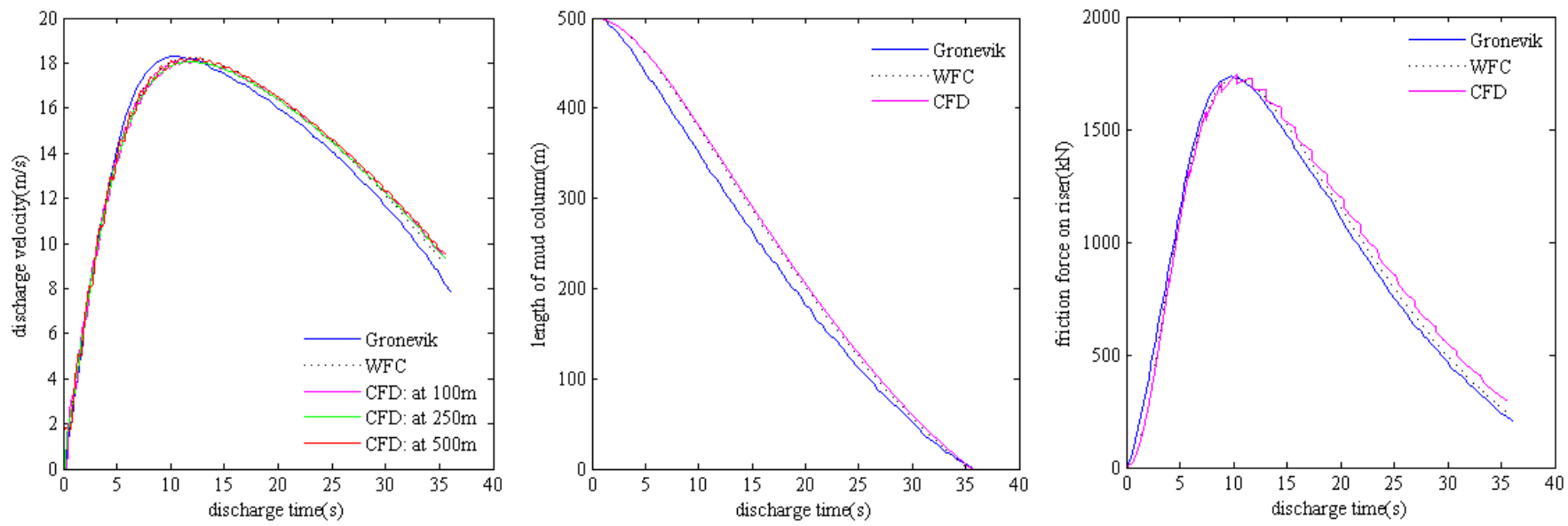

Fig. 3. Comparison with the results from Grønevik(2013)
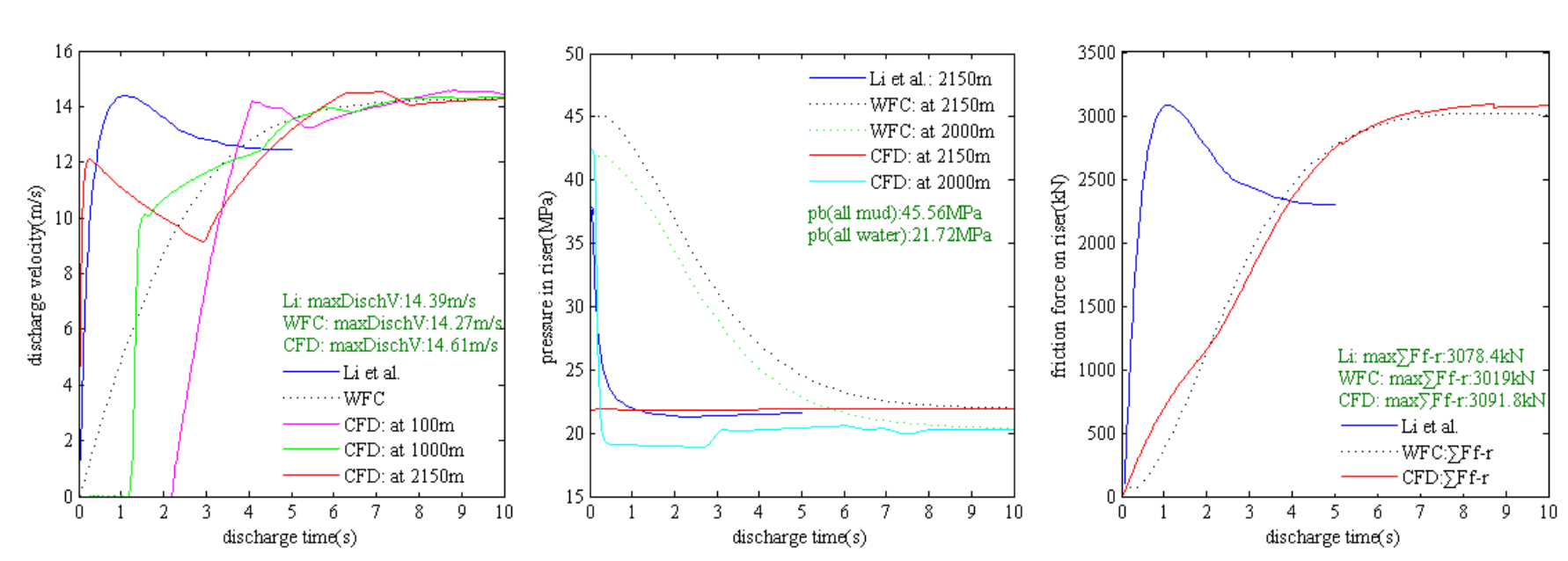

Fig. 4. Comparison with the results from Li et al.(2012)

\section{The third-order TVD Runge-Kutta scheme}

In order to obtain $U^{n+1}$ at next time step $t_{n+1}$ based on the given $U^{n}$ at time $t_{n}$, temporal discretization of Runge-Kutta scheme is implemented by three Euler steps:

$\left\{\begin{array}{l}U^{(1)}=U^{n}+\Delta t \cdot L\left(U^{n}\right) \\ U^{(2)}=\frac{3}{4} U^{n}+\frac{1}{4} U^{(1)}+\frac{1}{4} \Delta t \cdot L\left(U^{(1)}\right) \\ U^{n+1}=\frac{1}{3} U^{n}+\frac{2}{3} U^{2)}+\frac{2}{3} \Delta t \cdot L\left(U^{2)}\right)\end{array}\right.$

This scheme provides a high-order of temporal accuracy, and for CFL(Courant-Friedrichs-Lewy) number below 1 it shows very good numerical stability through its TVD properties. 

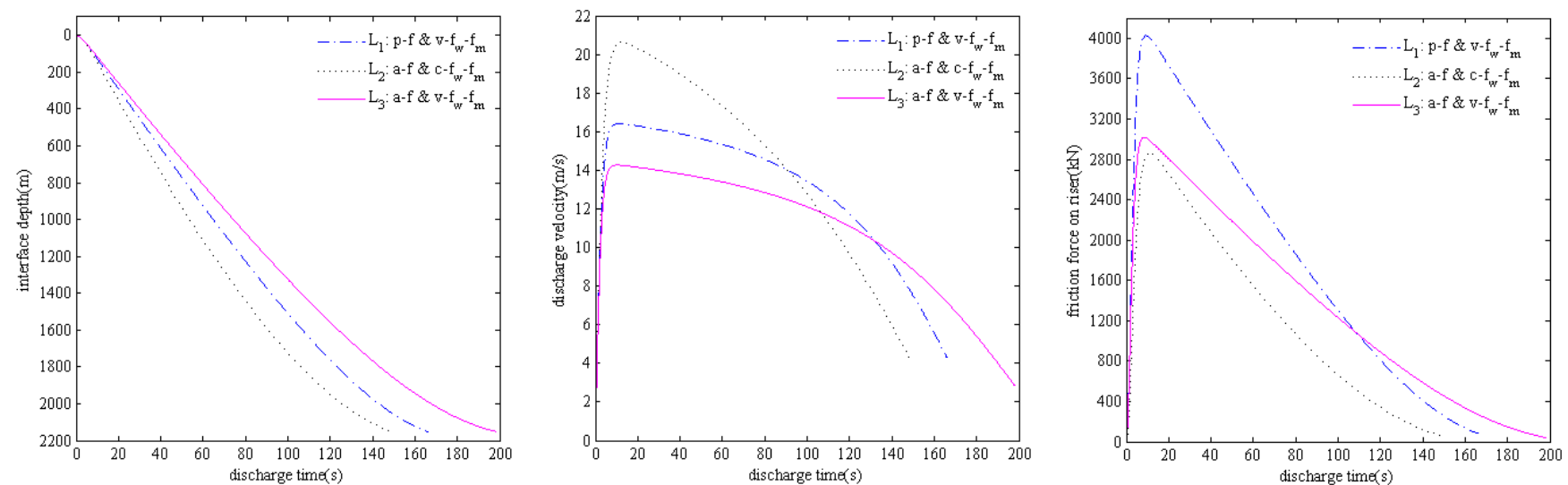

Fig. 5. Comparison with different flow channels and friction coefficients

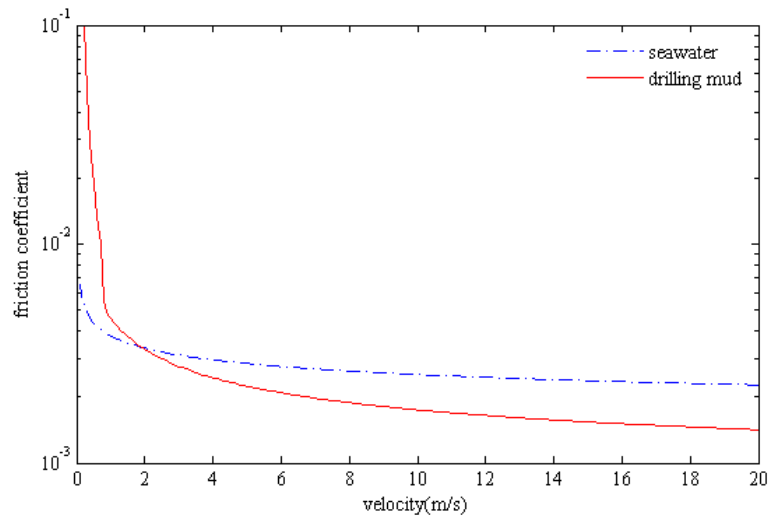

Fig. 6. Friction coefficients of seawater and drilling mud

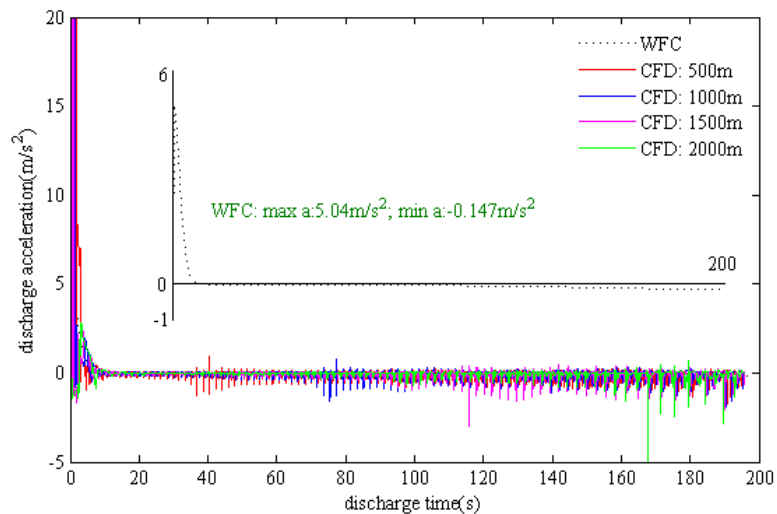

Fig. 8. Discharge acceleration

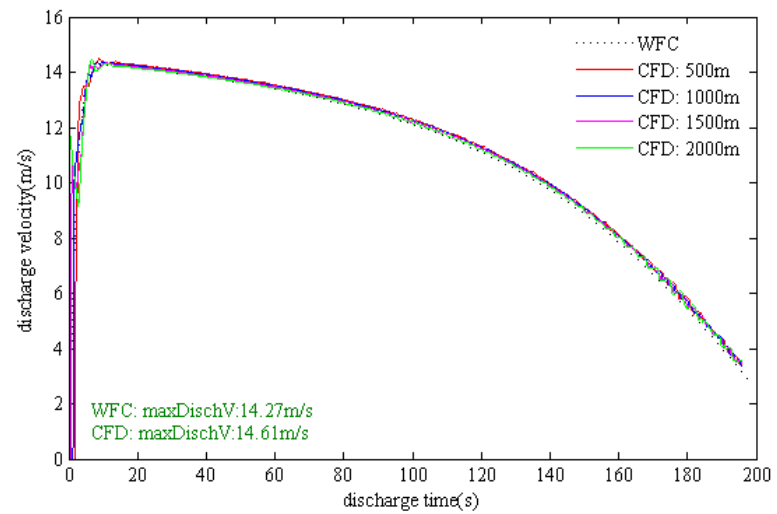

Fig. 10. Discharge velocity

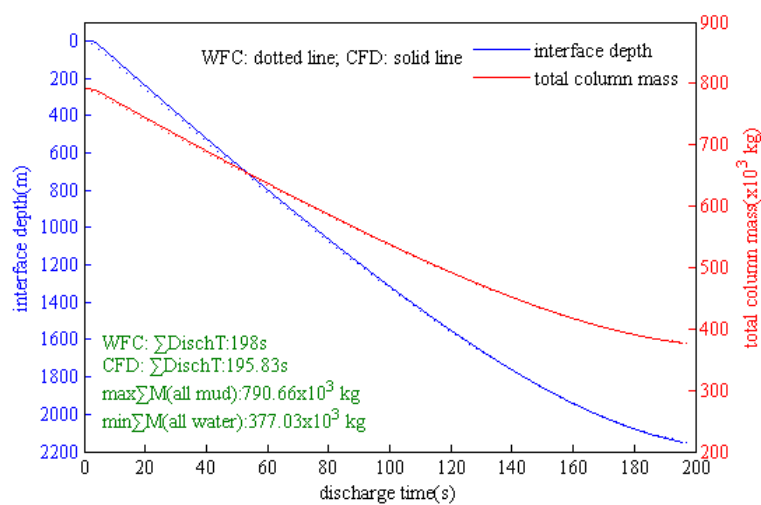

Fig. 7. Interface depth and total column mass

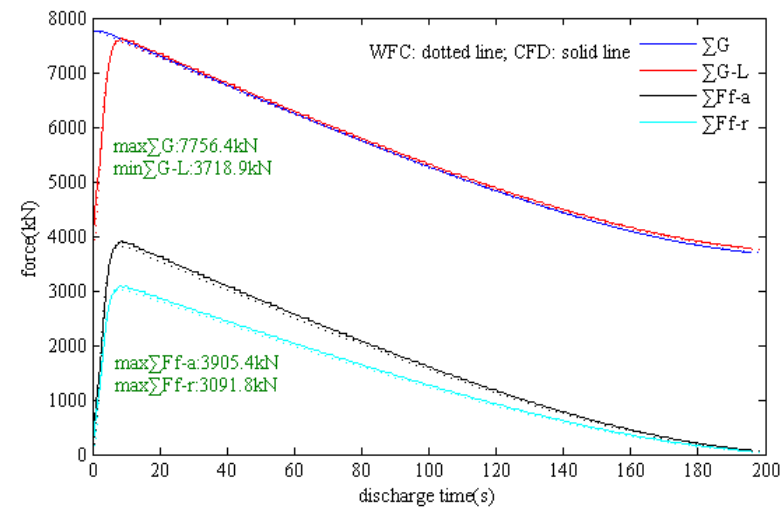

Fig. 9. Total column weight and total friction force

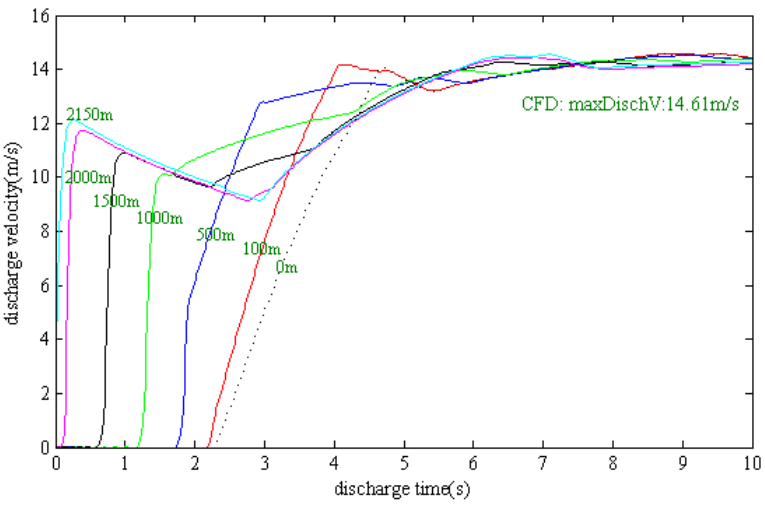

Fig. 11. Discharge velocity (the first 10 seconds) 


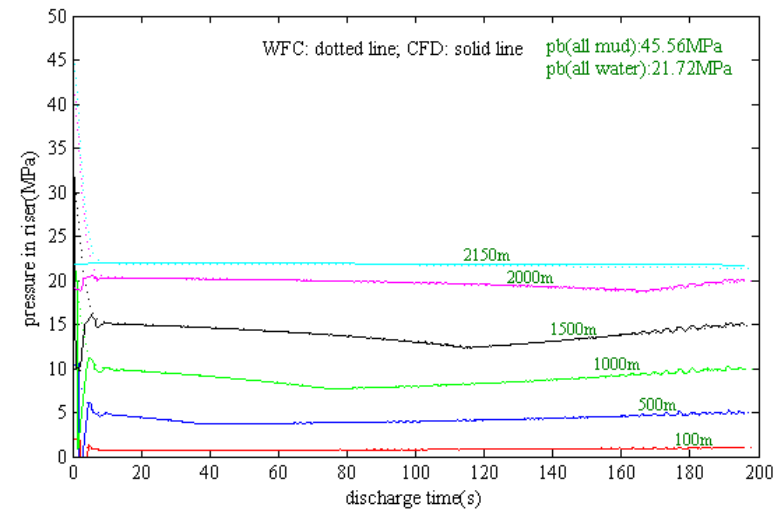

Fig. 12. Fluid pressure in riser annulus

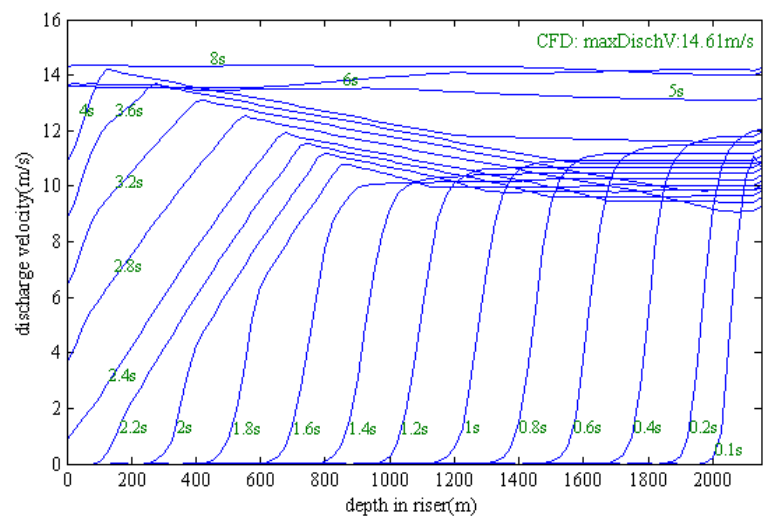

Fig. 14. Discharge velocity distribution with depth (the first 8 seconds)

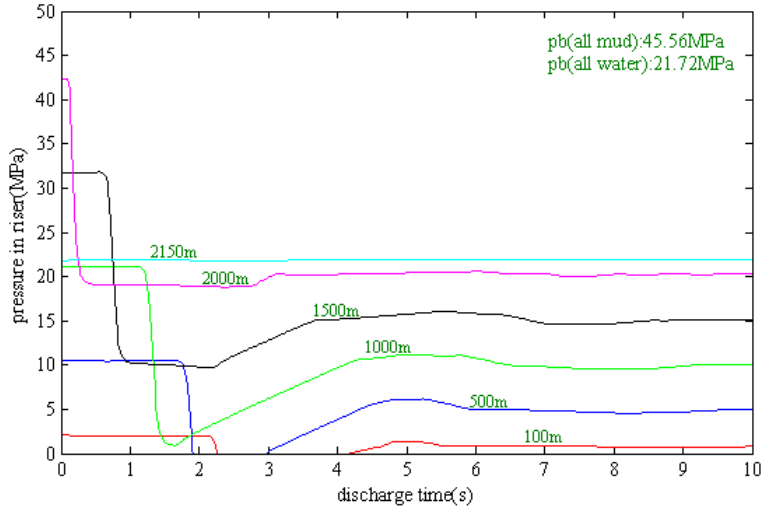

Fig. 13. Fluid pressure in riser annulus (the first 10 seconds)

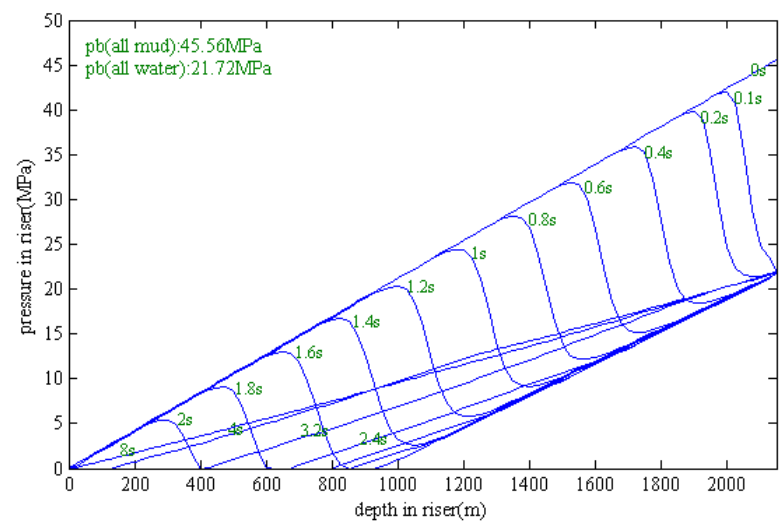

Fig. 15. Fluid pressure distribution with depth (the first 8 seconds)

Where: WFC/CFD-data from WFC method/CFD method; $\sum$ DischT-whole discharge time; $\sum \mathrm{M}$ (all mud/all water)- mass of total fluid column when annulus is sole mud/sole seawater; $\sum \mathrm{G} / \sum \mathrm{G}$-L-weight/weight after loss of whole fluid column; $\sum \mathrm{Ff}$-a/ $\sum \mathrm{Ff}$-r-total friction force of whole annulus/inner riser wall; $\mathrm{pb}$ (all mud/all water)-static pressure at riser bottom when whole annulus is sole mud/sole seawater. 